\title{
Resilience-based optimal design of water distribution network
}

\author{
C. R. Suribabu ${ }^{1}$
}

Received: 30 November 2016/Accepted: 4 April 2017/Published online: 11 April 2017

(c) The Author(s) 2017. This article is an open access publication

\begin{abstract}
Optimal design of water distribution network is generally aimed to minimize the capital cost of the investments on tanks, pipes, pumps, and other appurtenances. Minimizing the cost of pipes is usually considered as a prime objective as its proportion in capital cost of the water distribution system project is very high. However, minimizing the capital cost of the pipeline alone may result in economical network configuration, but it may not be a promising solution in terms of resilience point of view. Resilience of the water distribution network has been considered as one of the popular surrogate measures to address ability of network to withstand failure scenarios. To improve the resiliency of the network, the pipe network optimization can be performed with two objectives, namely minimizing the capital cost as first objective and maximizing resilience measure of the configuration as secondary objective. In the present work, these two objectives are combined as single objective and optimization problem is solved by differential evolution technique. The paper illustrates the procedure for normalizing the objective functions having distinct metrics. Two of the existing resilience indices and power efficiency are considered for optimal design of water distribution network. The proposed normalized objective function is found to be efficient under weighted method of handling multi-objective water distribution design problem. The numerical results of the design indicate the importance of sizing pipe telescopically along shortest path of flow to have enhanced resiliency indices.
\end{abstract}

C. R. Suribabu

suribabu@civil.sastra.edu

1 Centre for Advanced Research in Environment (CARE), School of Civil Engineering, SASTRA University, Thanjavur, Tamilnadu 613 401, India
Keywords Capital cost - Water distribution network . Resilience · Differential evolution

\section{Introduction}

An abundance of optimization techniques have been used for optimal design of water distribution with a main focus on cost minimization. Optimization of water distribution system either with cost or reliability or resilience as an objective requires a lot of computational effort and time and seldom converges directly to global optimal solution. As far as computational point of view, water distribution system problems are classified as highly complex and it is very unlikely that simple and efficient algorithms can be developed to obtain global optimal solution when design is subjected to numerous constraints and multi-objectiveness. Therefore, it is unfair to depend on a heuristic method which gives a reasonably good solution, if not the global one, with less computational effort. In many real-life problems, it is not necessary to obtain a global optimal solution. Any solution that satisfies constraints imposed to the problem and within a planned budget and close to optimal value can be treated as a satisfactory solution. Global minimum cost solution may not be right choice when the estimation of reliability of component or resilience of the system and uncertainty in the demand include errors and approximations (Loucks and Beek 2005). In general, resilience is defined as the ability of system to recover from its failure, setbacks, and to adapt well to change (e.g., climate change). Resilience of water distribution system strongly relates to the intrinsic capability of system to overcome failures. The water distribution network designed based on resilient point of view should have reduced failure probabilities and 
minimum or reduced failure consequences and able to recover quickly from failures. Recently, Cimellaro et al. (2015) defined resilience related to infrastructure facilities as the ability of the system to withstand, adapt to and rapidly recover from the effects of a disruptive event. The resilience of looped water distribution system can be enhanced by either providing redundancy at pipe capacity level or increasing component reliabilities or both. But such enhancement leads to increase in system cost. Thus, a tradeoff between these two options is indispensable for budgetconstrained design optimization. Generally, resilience measure depicts the additional capacity in the network and by increasing pipe sizes, resilience can be increased. However, it provides no quantification of performance under component failure condition as does the reliability measures. Least capital cost design for water distribution network using optimization technique usually results with a network configuration that can just satisfy the nodal demand under normal operating conditions with expected outlet pressure. The optimization model inherently selects the shortest possible path to the demand node and assigns a least dimensional pipe size to all en-route pipes to that node and other demand nodes too. There is lot of chances that the optimization model could have assigned smaller pipe sizes to almost all the redundant pipes in the looped network in view of cost minimization as a prime objective. Although loops ensure better connectivity to the nodes to the source, the undersized redundant pipes cannot satisfy the consumer's demand those who depend on these pipes directly and also during abnormal demand scenarios. Todini (2000) presented the concept of "resilience index" as an indicator of the functioning of a network under failure circumstances. Prasad and Park (2004) arrived at a quantity termed "network resilience" that describes redundancy of links in the network, thereby broadening resilience index. Fu et al. (2012) pointed out that there is no universal hydraulic performance measure available for design of water distribution network.

There are few attempts in which cost and resilience considered as twin objectives to optimize network with the aim of arriving least cost network with best possible performance. Although the formulation of multi-objective problem for water distribution network is simple, solving and arriving Pareto front is found to be challenging. Bolognesi et al. (2014) developed pseudo-front in the hydraulically feasible region in the multi-objective design of water distribution network by combining any two objectives (i.e., cost and performance measures such as deviation from minimum of pressure deficit, deviation of velocity and resilience measure). The formulated problem in three different objectives was solved by genetic heritage and stochastic evolution transmission (GHEST) algorithm. Creaco et al. (2014) formulated a multi-objective optimization problem for phasing design of water distribution system, which has two objectives, one as minimizing the costs of the upgrades converted into value at the initial time and other as maximizing the minimum pressure surplus over time. Ostfeld et al. (2014) used genetic algorithm for the multi-objective optimization of water distribution network considering cost and resilience index as twin objectives of the design and utilized split pipe size method, which assign more than one diameter for each pipe in the network. Piratla (2015) presented a three-objective optimization model to obtain various sustainable and resilient design alternatives. The trade-off among three objectives can help to identify most beneficial solution from Pareto optimal set of solutions. Recently, Wang et al. (2014) applied various multi-objective evolutionary algorithms to generate true Pareto front for two-objective model that belongs to optimal design of water distribution network. The various algorithms were tested using 12 benchmark networks available in the literature. The results of study indicate that non-dominated sorting genetic algorithm-II (NSGAII) is found to be most promising approach for solving two-objective water distribution network problems. Creaco et al. (2016) proposed three-objective optimization model to minimize the costs and maximize both the resilience and the loop-based diameter uniformity indexes. The study provides an improvement in the NSGA-II algorithm to overcome the difficulties that arises with more than two objectives. Most of the previous studies focused more on generating true Pareto front to the problem in an efficient manner. The aim of the present work is to provide a simple way to decision makers for obtaining best solution while considering both objectives simultaneously with or without due weightage to particular objective function. Further, it brings out the way in which the resiliency of network gets increased at the expense of cost in optimal manner. Hence, minimizing the cost and maximizing the resilience index are dealt in two different ways as a single objective optimization problem and illustrated with widely used Hanoi water distribution networks.

\section{Resilience indices}

Water distribution network is a prime hydraulic infrastructure to water supply system. Design of such system economically and efficiently is found to be one of the important research topics among researcher even in the present day. Todini (2000) perhaps is the first researcher who proposed a resilience index for water distribution network by relating nodal pressure and demand to address the intrinsic capability of system to overcome failures. Further, Todini (2000) pointed out that proposed resilience 
measure is a surrogate to the reliability of the system. The calculation of reliability of the system requires pipe failure data and its consequence in the supplying water to the consumers. Although the resilient measure does not take into account of the failure data, network designed based on resilient measure can sustain at failure of its components. It is clear that resilient design of water distribution network is strongly related to the intrinsic capability of system that will have reduced failure probabilities and minimum or reduced failure consequences and able to recover quickly from failure. Resilience of the system is also viewed as a measure of capability of the system to absorb the shocks or to perform under perturbation. Howard and Bartram (2010) defined the resilience of a piped water supply as a function of the resilience of individual components of the system, namely, the source, treatment, and distribution through primary, secondary, and tertiary pipes and in system storage infrastructure. Wu et al. (2011) developed a surplus power factor as resilience measure for optimization of water main transmission system. Yazdani and Jeffray (2012) used robustness and redundancy to define the resilience of water distribution system. Liu et al. (2012) provided an overall definition for resilience as capacity of water resources system to maintain it essential functions as before during an event of unexpected stresses and disturbances. Resilience-based design of various infrastructures facilities is also gaining importance. Bruneau et al. (2003) presented a conceptual framework for defining seismic resilience by integrating four dimensions of community resilience, namely technical, organization, social, and economic which can be used to quantify measures of resilience for various types of physical and organizational systems. Wang et al. (2009) illustrated that the resilient infrastructure is one that shows (a) reduced failure probabilities, (b) reduced consequence of failure, and (c) reduced time to recovery. Cimellaro et al. (2010) presented concepts of disaster resilience and procedure for its quantitative evaluation and the same is illustrated using two examples through hospital network subjected to earthquakes. Saldarriaga et al. (2010) presented a new prioritization approach based on the resilience index and dissipated hydraulic power in the pipe to arrive hydraulically and economically near optimal solution to the rehabilitation problems. Pandit and Crittenden (2012) proposed an index to address the resilience using six network attributes. Davis (2014) demonstrated the relation between resilience and water system serviceability, and dependence of community resilience on water system resilience and defined five water service categories namely water delivery, quality, quantity, fire protection, and functionality to address the water system resilience. These five service categories were applied to Los Angeles water system after earthquake condition to find the means of improving the system for quick restoration. Cimellaro et al. (2015) proposed a new resilience index for water distribution network by combining three issues based on water availability to the consumer, storage level of water tank, and water quality. The developed index was applied to Calascibetta town water supply system located in Italy under different disruptive scenarios.

Todini (2000) formulated resilience index (RI) for water distribution network as the ratio between the sum of residual power of all the nodes and the sum of potential residual power of all the nodes:

$\mathrm{RI}=\frac{\sum_{j=1}^{N} q_{i\left(h_{\mathrm{avl}}, j-h_{\text {min } \mathrm{j}}\right)}}{\left(\sum_{i=1}^{R} Q_{r} h_{\mathrm{res}, i}+\sum_{b=1}^{B} \frac{P_{b}}{v}\right)-\sum_{j=1}^{N} q_{j} h_{\mathrm{min}, \mathrm{j}}}$,

where $q_{i}$ represents demand at node $i ; h_{\mathrm{avl}, j}$, available pressure head at node $j ; h_{\min , j}$, minimum pressure head at node $j ; Q_{\mathrm{r}}$, flow from reservoir $i ; h_{\mathrm{res}, i}$, sum of reservoir elevation and its water level of reservoir $i ; P_{b}$, capacity of pump $b$; and $v$ is the specific weight of the liquid.

Jayaram and Srinivasan (2008) formulated another resilience index called as modified resilience index (MRI) as given below with small modification over Todini's (2000) resilience. It is defined as the ratio between the sum of residual power of all the nodes and the sum of minimum power required to all the nodes:

MRI $=\frac{\sum_{j=1}^{N} q_{i\left(h_{\mathrm{avl}}, j-h_{\mathrm{min}, \mathrm{j}}\right)}}{\sum_{j=1}^{N} q_{j} h_{\mathrm{min}, \mathrm{j}}}$.

It is to be noted that Todini (2000) resilience index theoretically varies between 0 and 1 (poor and good). But, in the case of modified resilience index, its value can exceed one. Although both indices quantify intrinsic capability of the system, it is very difficult to fix a predefined value in case of taking resilience as constraint to the optimization model. The nodes near to source will have more surplus power than the critical nodes. The critical node is one which will not have sufficient surplus power to support supply of demand during abnormal operation condition. Generally, utilization of surplus power of the nodes located near to the source will certainly affect the supply at critical nodes. Hence, minimum surplus power at critical nodes should be considered in quantifying the intrinsic capability of the system. The major advantage of Todini and Jayaram and Srinivasan's resilience indices is that it does not involve any statistical considerations on failures. Resilience of the network can be improved only if the network is designed to have higher value of indices at the planning and design stage itself. Todini (2000) pointed out that resilience of the system can be enhanced only through more even distribution of flow among all the pipes rather than allowing flow concentrically in a spanning tree. Suribabu and Neelakantan (2012) formulated a simple 
performance index called power efficiency based on power availability at the source and power delivered at the demand nodes. It is defined as the ratio between sum of the power delivered at the demand nodes and total power available at the source reservoirs while satisfying the constraints on the nodal demands and nodal heads. Power efficiency indicator takes the total power available in denominator in the expression; the ratio is found to be simpler to visualize than other power-based performance measures:

$\eta=\frac{\sum_{j=1}^{N} Q_{j} h_{j}}{\sum_{i=1}^{R} Q_{i} h_{\mathrm{res}, i}} \times 100$,

where $Q_{\mathrm{s}}$ and $h_{\text {res }}$ are the discharge and the head, respectively, available at reservoir $i$; $R$, the number of reservoirs; $Q_{j}$ and $h_{j}$, the demand and the available head at node $j$; and $N$ is the number of demand nodes. The power efficiency $\eta$ theoretically ranges between 0 and $100 \%$ (poor and good). Recently, Suribabu et al. (2016) illustrated methods for improving the resilience of existing water distribution network, and analysis of the results has shown that marginal increase in cost can fetch significant increase in the resilience of the network.

It is evident that selection of higher dimension pipe than actually required as far as economical point of view can increase the resilience of network. Hence, minimizing the cost and maximizing the resilience of the network will be more appropriate if the network is to be designed based on not only economical point of view but also resilience of the system. Reliability of the system can be increased by providing stand-by pumps and storage facilities if water source is reliable. But in the case of components such as pipes and valves, economical and technical feasibility of providing stand-by pipes and valves is herculean task. It is difficult and too costly to have parallel pipe for each pipe or even for some critical pipes. In case of possible situations to have parallel pipes, the house connections taken from the main pipe cannot be shifted to parallel pipe at the time of failure or abnormal operating condition. Hence, it is wise to size the pipe to have additional capacity at the design stage itself. The question of extending capacity of pipe can be achieved by formulating design problem as a multi-objective problem. This paper uses these three existing measures which address the resilience of water distribution network.

\section{Weighted diameter}

The network optimized considering resilience index usually end up with higher size with respect to optimal solution without consideration of resilience measure. Hence, the optimal result obtained by considering various resilience measures can be distinct to each other. To make common comparison and better understanding of pipe size configuration of the network, the calculation of weighted diameter based on each pipe diameter and its length is proposed as follows:

$d_{\mathrm{w}}=\frac{\sum_{i=1}^{n} L_{i} D_{i}}{\sum_{i=1}^{n} L_{i}}$

where $L_{i}$ is the length of the pipe $i$ and $D_{i}$ is the diameter of the pipe $i$.

This simple expression will be useful to assess the increase in overall pipe size due to increase in overall cost. Further, it provides an idea about how the additional space is created to increase the resilience of the network.

\section{Optimization model}

Objective function of the model is defined as minimization of network cost per unit resilience measure.

Min $C=\mathrm{PC} / \mathrm{RM}$,

where $\mathrm{PC}=\sum_{j=1}^{\mathrm{nl}} P_{d(j)} \cdot l_{j}$ is the pipe cost of the network; $\mathrm{nl}$, the number of links in the network; $d(j)$, the diameter selected for link $j ; P_{d(j)}$, the pipe cost per unit length for the link $j$ with diameter $d(j)$; and $l_{j}$ is the length of link. RM is a resilience measure according to selected index from expressions (1), (2), and (3).

This objective function needs to be minimized subject to a set of hydraulic constraints as follows:

The continuity of flow each nodal point is expressed in the mathematical form as

$\sum_{i \in \text { in }, n} Q_{i}=\sum_{j \in \text { out }, n} Q_{j}+\mathrm{ND}_{n} \quad \forall n \in \mathrm{NN}$,

where $Q$ is the pipe flow; $\mathrm{ND}_{n}$, the demand at node $n$; in, $n$, the set of pipes entering to the node $n$; and out, $n$, the set of pipes emerging from node $n$; and $\mathrm{NN}$ is the node set.

The total head loss around the closed path (loop) should be equal to zero or the head loss along a path between nodes should be equal to the difference in nodal head:

$\sum_{i \in \operatorname{loop} p} \mathrm{hf}_{i}=\Delta H, \quad \forall p \in \mathrm{NL}$,

where $\mathrm{hf}_{i}$ is the head loss due to friction in pipe $i$; NL, the loop set; $\Delta H$, the difference between nodal heads at both ends; and $\Delta H=0$, if the path is closed.

The Hazen-Williams head loss equation for pipe $i$ of connecting nodes $j$ and $k$ :

$h_{j}-h_{k}=\operatorname{hf}_{i}=\frac{\alpha L_{i} Q_{i}\left|Q_{i}\right|^{0.852}}{C_{\mathrm{HW}, i}^{1.852} D_{j}^{4.87}} \quad \forall j \in N P$, 
where NP is the number of pipes; $C_{\mathrm{HW}}$, the Hazen-Williams coefficient; $D_{i}$, the diameter of the pipe $i ; L_{i}$,the length of the pipe $i ; \alpha$ is the conversion factor which depend on the units, different values of $\alpha$ are found in the literature-as low as 10.4516 to as high as 10.9031 (Savic and Walters 1997).

The pressure head in all the nodes should be greater than prescribed minimum pressure head:

$h_{j} \geq h_{\min , \mathrm{j}}$,

where $\mathrm{h}_{j}$ is the pressure head at node, $j$ and $h_{\min , j}$ is the minimum required pressure head.

The diameter of the pipes should be selected from listed commercial size:

$D_{j}=[D], \quad \forall i \in \mathrm{NP}$

\section{Normalized objective function}

The resilience-based optimal design of the network can also be performed using proposed normalized objective function as given below in addition to optimizing network with cost per resilience measure. A simple way to evaluate both objectives is to combine both objectives through appropriate weights. The selection of appropriate weights can also be done based on the importance predicted by the water engineer or decision maker. This can be implemented by considering either equal weights for each objective function or different weightage to each objective function according to the priority and important that decision maker considers. Whenever weighted approach is used, it is essential to normalize each objective function so that different units of objective functions value are scaled down uniquely to the problem. The following formula presents weighted sum of normalized cost and resilience measure of network as a single objective function:

$Z_{\min }=w \frac{C-C_{\min }}{C_{\max }-C_{\min }}+(1-w) \frac{\mathrm{RM}_{\max }-\mathrm{RM}_{\min }}{\mathrm{RM}-\mathrm{RM}_{\min }}$,

where $C$ is the cost of the network; $C_{\min }$, optimal cost of the network; $C_{\max }$, maximum cost of the network; $W$, weightage factor; RM, resilience measure of the network; $\mathrm{RM}_{\text {min }}$, resilience measure of the network corresponding to the optimal solution; $\mathrm{RM}_{\max }$ is the resilience measure of the network corresponding to the maximum cost solution.

Minimum cost solution denotes the optimal solution obtained without considering resilience measure and $\mathrm{RM}_{\min }$ is its resilience measure value. $C_{\max }$ is obtained by configuring all the pipes of network with maximum commercial diameter. $\mathrm{RM}_{\max }$ denotes the resilience measure obtained for $C_{\max }$ network.

\section{Differential evolution algorithm}

Differential evolution (DE) algorithm is a search-based stochastic optimization algorithm introduced by Storn and Price (1995) for solving complex continuous optimization problems as an improvement over genetic algorithm. DE algorithm uses population-based solution exploration with the help of crossover, mutation, and selection operators. It explores the best candidate solutions iteratively until the stopping criterion is reached. Differential evolution algorithm requires an initial population containing individuals or vectors (candidate solutions) that can be generated randomly. The fitness value of each candidate solution obtained from the initial population is calculated according to the chosen objective function. Two candidate solutions are selected randomly from the population and the vector difference between them is calculated and its weighted value is calculated by multiplying factor called mutation $(0-1)$ and resulting weighted vector is added with the third randomly selected candidate solution which needs to be selected from the population other than earlier selected two candidate solutions. The new candidate solution so obtained from above process is called as noisy vector. This noisy vector is now subjected to crossover process with a target vector selected randomly from the population. The candidate solution obtained at the end of crossover process is called trial vector. The vector having better fitness between trial and target vectors is considered as a candidate solution to the next generation. The number of candidate solutions for next generation for chosen population size is obtained by repeating the above-mentioned procedure number of times equal to the population size. The entire process is repeated either by predefined number of generations or until specified termination criteria is achieved. The stepwise procedure is illustrated as follows:

1. Initial candidate solutions are generated randomly for chosen population size (pop_size) to form initial population and accounting this as a first generation $(G=1)$. The expression for creating random solution is as follows:

$d_{i, j}^{0}=d_{j}^{(L)}+r_{i, j}^{G}\left(d_{j}^{(U)}-d_{j}^{(L)}\right), \quad \forall i=1$ to $s$,

$\forall j=1$ to $n$,

where $r_{i, j}^{G}$ denotes a uniformly distributed random value within the range from 0.0 to $1.0 ; d_{j}^{(U)}$ and $d_{j}^{(L)}$ are upper and lower limits of variable $d_{j}$; and $s$ and $n$ denotes population size and number of variables, respectively.

2. In the next step, weighted vector is calculated by multiply mutation factor $F$ with differential vector 
obtained by finding the difference between two randomly selected vectors from population:

$w_{j}^{G}=F *\left(d_{A, j}^{G}-d_{B, j}^{G}\right) \quad \forall_{j}=1$ to $n$.

The weighing factor $(F)$ is usually selected between 0.4 and 1.0.3. The population of trial vectors $P^{(G+1)}$ is generated as follows:

$d_{i, j}^{G+1}=\left\{\begin{array}{ll}d_{C, j}^{(G)}+w_{j}^{G} & \text { if } r_{i, j} \leq C_{r} ; \quad \forall j=1 \text { to } n \\ d_{i, j}^{(G)} & \text { otherwise }\end{array}\right.$,

where $i=1, \ldots$,pop_size, $A \in[1, \ldots$,pop_size $], B \in[1$, ...,pop_size], $C \in[1, \ldots$,pop_size $], A \neq B \neq C \neq i, C_{r} \in$ [0 to 1$], F \in[0$ to 1$], r \in[0$ to 1$]$.

$C_{\mathrm{r}}$ is crossover constant, which assists for differential perturbation in order to select the pipe diameter either from noisy vector or target vector to form a new population for next generation.

4. The population of next generation $P^{(G+1)}$ is created as follows (Selection):

$x_{i, j}^{G+1}=\left\{\begin{array}{ll}d_{i, j}^{(G+1)} & \text { if } f\left(d_{i}^{(G+1)}\right) \leq f\left(d_{i}^{(G)}\right) \\ d_{i, j}^{(G)} & \text { otherwise }\end{array}\right.$,

where $f\left(d_{i}^{(G)}\right)$ represents the cost of $i$ th individual in $G$ th generation.

Further details on implementation of DE to the design of water distribution are available in Suribabu (2010). The recent studies on optimal design of water distribution network using DE algorithm (Suribabu 2010; Vasan and Simonovic 2010; Zheng et al. 2011a, b, 2012a, b, Dong et al. 2012; Marchi et al. 2014) have shown as a most promising population-based stochastic search technique. In the present study, the combined computer coding of DE algorithm and functions of EPANET (Rossman 2000) Toolkits for hydraulic simulation has been written in Visual Basic language.

\section{Illustration with Hanoi network}

The most widely used benchmark water distribution network called Hanoi city water distribution network is taken for the present study to illustrate resilient-based optimal design of water distribution (Fig. 1). This network consists of 32 nodes, 34 pipes, and a reservoir. Fujiwara and Khang $(1990,1991)$ presented the complete data for design of the network and same is presented through Tables 1 and 2 . Hazen-Williams coefficient and minimum nodal pressure head are defined as 130 for all the pipes and $30 \mathrm{~m}$ for each demand node, respectively.

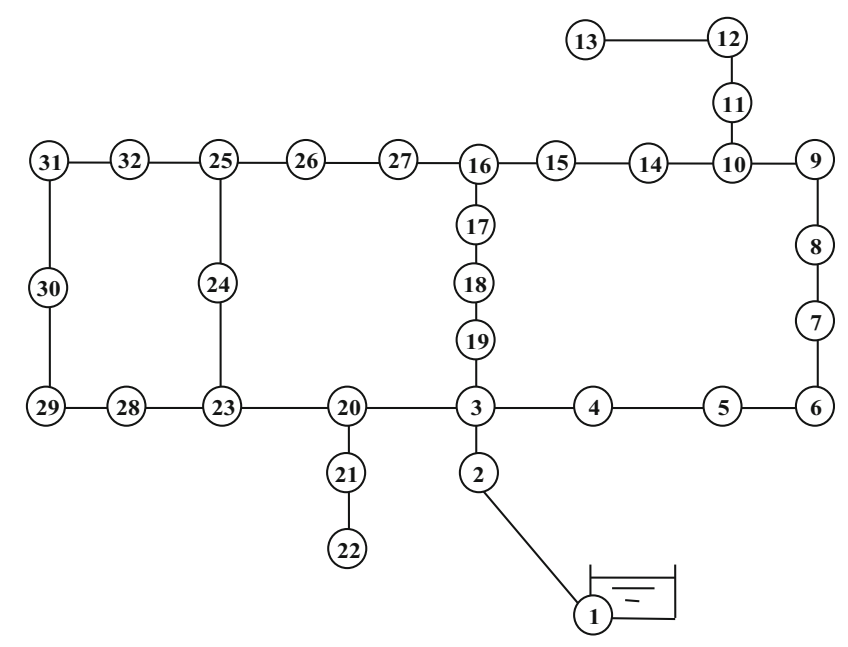

Fig. 1 Layout of Hanoi network

\section{Results and discussion}

The example network is optimized for single objective function which is defined as cost per resilience index. Optimization is performed for three different resilience measures. Table 3 provides the optimal solution obtained for various resilience measures. Table 3 shows that network cost is higher while considering resilience measure proposed by Todini (2000), whereas solution based on other two measures, namely modified resilience measure proposed by Jayaram and Srinivasan (2008) and power efficiency measure proposed by Suribabu and Neelakantan (2012), are resulted with same cost solution and found to be lesser than the solution based on Todini's resilience measure. The optimal values of RI, MRI, and PE corresponding to objective function cost per resilience measure are 0.317 , 1.675 and 0.503 , respectively. The characteristic of MRI and PE is found to be similar while arriving optimal solution for the presented network problem. The optimal cost of network without consideration of RM as reported in the literature is $\$ 6,081,087$ which is lower than the Cost/ RM. This clearly indicates that the least cost network without consideration of resilience measures can certainly be a less resilience as its value on all three considered resilience measures is found to be less than other two solutions. The proposed first model directly results a single solution which could be helpful to the decision makers as it acts a pivot to the Pareto optimal solutions in case of solving as a multi-objective problem. The weighted diameter value gives an overall picture in terms of the geometry of pipe that network has. The least cost solution (i.e., \$6,081,087) has a weighted diameter of $655.63 \mathrm{~mm}$ and other two solutions are having higher values according to its cost. The increase in diameter of certain pipes in other two solutions brings down the head loss due to friction and 
Table 1 Node and link data for Hanoi network

\begin{tabular}{|c|c|c|c|c|}
\hline Node No. & Demand $\left(\mathrm{m}^{3} / \mathrm{h}\right)$ & Link index & Arc & Length (m) \\
\hline 1 & $-19,940$ & 1 & $(1,2)$ & 100 \\
\hline 2 & 890 & 2 & $(2,3)$ & 1350 \\
\hline 3 & 850 & 3 & $(3,4)$ & 900 \\
\hline 4 & 130 & 4 & $(4,5)$ & 1150 \\
\hline 5 & 725 & 5 & $(5,6)$ & 1450 \\
\hline 6 & 1005 & 6 & $(6,7)$ & 450 \\
\hline 7 & 1350 & 7 & $(7,8)$ & 850 \\
\hline 8 & 550 & 8 & $(8,9)$ & 850 \\
\hline 9 & 525 & 9 & $(9,10)$ & 800 \\
\hline 10 & 525 & 10 & $(10,11)$ & 950 \\
\hline 11 & 500 & 11 & $(11,12)$ & 1200 \\
\hline 12 & 560 & 12 & $(12,13)$ & 3500 \\
\hline 13 & 940 & 13 & $(10,14)$ & 800 \\
\hline 14 & 615 & 14 & $(14,15)$ & 500 \\
\hline 15 & 280 & 15 & $(15,16)$ & 550 \\
\hline 16 & 310 & 16 & $(16,17)$ & 2730 \\
\hline 17 & 865 & 17 & $(17,18)$ & 1750 \\
\hline 18 & 1345 & 18 & $(18,19)$ & 800 \\
\hline 19 & 60 & 19 & $(19,3)$ & 400 \\
\hline 20 & 1275 & 20 & $(3,20)$ & 2200 \\
\hline 21 & 930 & 21 & $(20,21)$ & 1500 \\
\hline 22 & 485 & 22 & $(21,22)$ & 500 \\
\hline 23 & 1045 & 23 & $(20,23)$ & 2650 \\
\hline 24 & 820 & 24 & $(23,24)$ & 1230 \\
\hline 25 & 170 & 25 & $(24,25)$ & 1300 \\
\hline 26 & 900 & 26 & $(25,26)$ & 850 \\
\hline 27 & 370 & 27 & $(26,27)$ & 300 \\
\hline 28 & 290 & 28 & $(27,16)$ & 750 \\
\hline 29 & 360 & 29 & $(23,28)$ & 1500 \\
\hline 30 & 360 & 30 & $(28,29)$ & 2000 \\
\hline 31 & 105 & 31 & $(29,30)$ & 1600 \\
\hline \multirow[t]{3}{*}{32} & 805 & 32 & $(30,31)$ & 150 \\
\hline & & 33 & $(31,32)$ & 860 \\
\hline & & 34 & $(32,25)$ & 950 \\
\hline
\end{tabular}

Table 2 Cost data for pipes for Hanoi network

\begin{tabular}{lcc}
\hline Diameter (in) & Diameter $(\mathrm{mm})$ & Cost (units) \\
\hline 12 & 304.8 & 45.73 \\
16 & 406.4 & 70.40 \\
20 & 508.0 & 98.38 \\
24 & 609.6 & 129.333 \\
30 & 762.0 & 180.8 \\
40 & 1016.0 & 278.3 \\
\hline
\end{tabular}

that helps to increase its resilience measure. But, at the same time, such an increase should not lead uneconomical condition for particular value of RM. For example, if two
Table 3 Optimal solutions for the network based on first objective function

\begin{tabular}{|c|c|c|c|}
\hline Pipe ID & Cost/RI & $\begin{array}{l}\text { Cost/MRI and } \\
\operatorname{cost} / \eta\end{array}$ & $\begin{array}{l}\text { Optimal } \\
\text { cost }\end{array}$ \\
\hline 1 & 1016 & 1016 & 1016 \\
\hline 2 & 1016 & 1016 & 1016 \\
\hline 3 & 1016 & 1016 & 1016 \\
\hline 4 & 1016 & 1016 & 1016 \\
\hline 5 & 1016 & 1016 & 1016 \\
\hline 6 & 1016 & 1016 & 1016 \\
\hline 7 & 762 & 762 & 1016 \\
\hline 8 & 762 & 762 & 1016 \\
\hline 9 & 609.6 & 762 & 1016 \\
\hline 10 & 762 & 762 & 762 \\
\hline 11 & 762 & 762 & 609.6 \\
\hline 12 & 508 & 508 & 609.6 \\
\hline 13 & 609.6 & 406.4 & 508 \\
\hline 14 & 762 & 508 & 406.4 \\
\hline 15 & 762 & 609.6 & 304.8 \\
\hline 16 & 1016 & 1016 & 304.8 \\
\hline 17 & 1016 & 1016 & 406.4 \\
\hline 18 & 1016 & 1016 & 609.6 \\
\hline 19 & 1016 & 1016 & 508 \\
\hline 20 & 1016 & 1016 & 1016 \\
\hline 21 & 609.6 & 609.6 & 508 \\
\hline 22 & 406.4 & 406.4 & 304.8 \\
\hline 23 & 1016 & 762 & 1016 \\
\hline 24 & 762 & 508 & 762 \\
\hline 25 & 609.6 & 304.8 & 762 \\
\hline 26 & 304.8 & 508 & 508 \\
\hline 27 & 609.6 & 762 & 304.8 \\
\hline 28 & 609.6 & 762 & 304.8 \\
\hline 29 & 406.4 & 406.4 & 406.4 \\
\hline 30 & 304.8 & 304.8 & 304.8 \\
\hline 31 & 304.8 & 304.8 & 304.8 \\
\hline 32 & 406.4 & 304.8 & 406.4 \\
\hline 33 & 406.4 & 406.4 & 406.4 \\
\hline 34 & 609.6 & 508 & 609.6 \\
\hline Cost \$ & $7,128,424.4$ & $6,650,114.42$ & $6,081,087$ \\
\hline $\begin{array}{l}\text { Weighted diameter } \\
\quad(\mathrm{mm})\end{array}$ & 738.58 & 702.76 & 655.63 \\
\hline RI & 0.317 & 0.289 & 0.192 \\
\hline MRI & 1.739 & 1.675 & 1.447 \\
\hline PE & 0.522 & 0.503 & 0.434 \\
\hline
\end{tabular}

solutions with similar RM with meager difference between them can have different weighted diameters due to distinct selection of path. Hence, this also can be utilized as an another measure to identify the better solution. The selection of diameter to the pipes in series for particular route should appear telescopic decreasing size from source to 
sink. The pipe ID 1-6 of the solutions based on RM has $1016 \mathrm{~mm}$ and in case of least cost solution, it is from Pipe ID 1-9. In another route, i.e., pipe ID 16-20 too has $1016 \mathrm{~mm}$ diameter for solutions based on RM, but which is not the case for least cost solution. This clearly depicts that the network optimized based on cost alone could result distinct configuration than with the RM. While comparing the optimized solutions based on RM, the selected diameter along major flow carrying routes appears to be similar. On comparison of two RM-based solutions, pressure head in 26 nodes of first solution is having higher value than the second solution obtained based on MRI/PE. Thus, only three nodes are having lesser pressure and two nodes with same pressure values. It is evident that $7.19 \%$ increase in cost makes around $9.69 \%$ increase in RI and $3.82 \%$ in MRI and $3.78 \%$ in PE. With respect to optimal solution, $9.36 \%$ increase in cost makes $50.52 \%$ increase in RI, $15.76 \%$ in MRI and $15.90 \%$ in PE, and in case of $17.22 \%$ increase in cost produces $65.10 \%$ increase in RI, $20.18 \%$ in MRI, and $20.28 \%$ in PE. Above analysis confirms that increase in the cost of the network optimally can bring considerable increase in RM. Most of the resilience measures proposed are related with pressure head at delivery nodes and if the service pressure head is higher than desired, then such an index value will be higher. This can be achieved through the sizing of the pipe. The pipe size will be higher than actually required as per economical point alone. Thus, higher pipes are having lesser head loss for given flow than the smaller size. Hence, increase in the pipe size always brings increase in pressure head and increases resilience measures.

The same network is optimized with weighted objective function as shown in Eq. (11). The network is optimized for all three RM and Pareto curves that are obtained by assigning different weights with weightage as increment of 0.05 . In the normalized objective function, the denominator part of second term of equation should be a positive value and should be greater than $\mathrm{RM}_{\min }$ value. $\mathrm{RM}$ value of infeasible solution will be less than $\mathrm{RM}_{\min }$. If it is used as it is then second term becomes negative and such a solution will be selected as a best solution. To avoid and eliminate such a condition, the RM value of such solution is increased 0.000001 from $\mathrm{RM}_{\text {min }}$ so that difference between $\mathrm{RM}$ and $\mathrm{RM}_{\min }$ will be always equal to 0.000001 . For each weightage pair [i.e., $w$ and $(1-w)$ ], ten trial runs are carried out by changing random seed value. The least value obtained as per normalized objective function is selected for every pair of weights and Pareto curve is generated and presented in Figs. 2, 3, 4. It can be seen from each curve that slope of the curve is steep between 6.25 and 7.5 millions and mild slope appears above 7.5 millions. This depicts that there is a drastically improvement of the network performance when the network is designed optimally

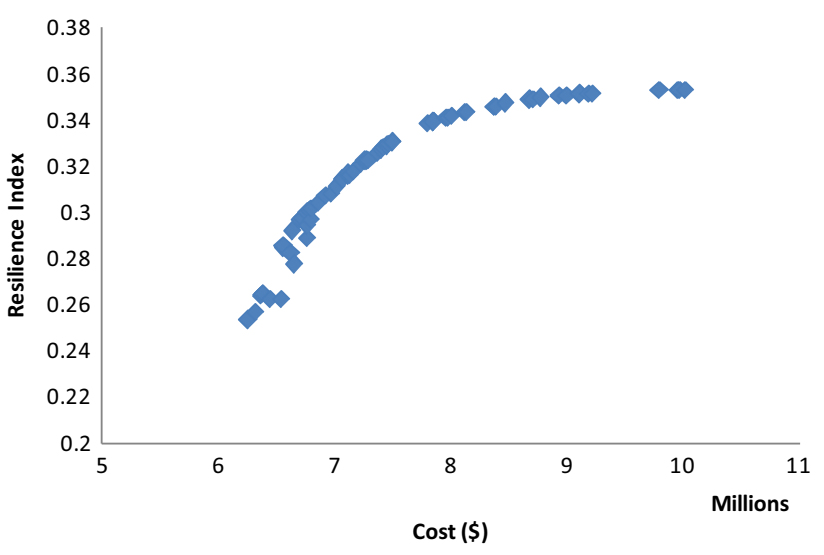

Fig. 2 Resilience index vs Cost Pareto curve

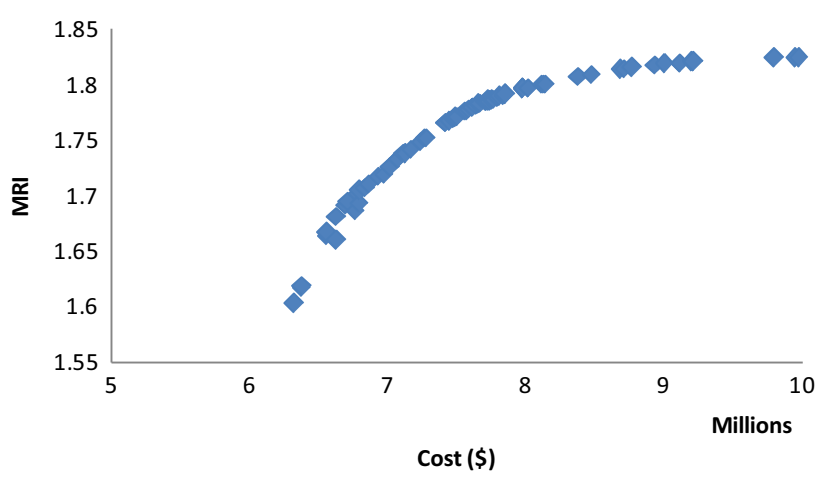

Fig. 3 Modified Resilience index vs Cost Pareto curve

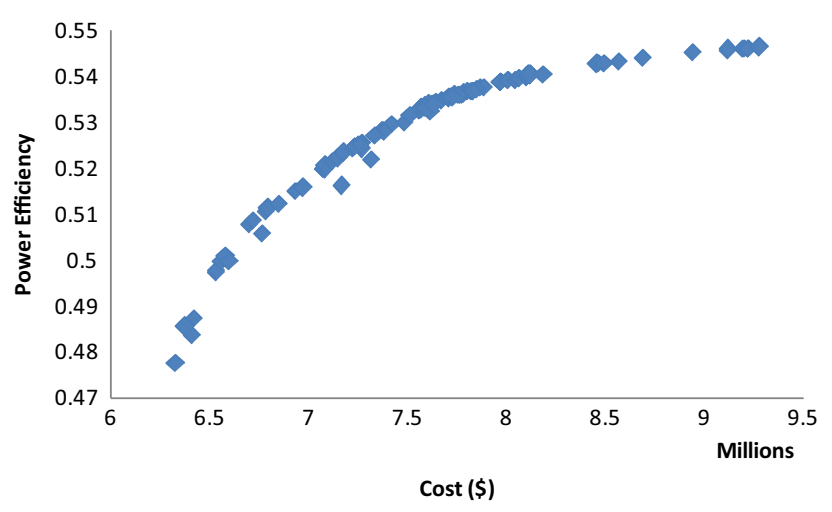

Fig. 4 Power Efficiency vs Cost Pareto curve

within the range of cost from 6.25 to 7.5 millions. It is found that the proposed normalized objective function is fully capable of arriving an optimal solution with minimum number of trial runs under population size of 50 and maximum number of generation 2000. The success rate of getting optimal solution irrespective of RM used for optimization is around 50\%. The main observation in the solution configuration is that the optimized solution at the higher level resilience measures follows dimensioning the pipe telescopically along the shortest path to the demand 
nodes. It is considered generally that transporting demand along shortest path provides cheapest mode of transport (Kadu et al. 2008). With an increase in solution cost, the diameter of the pipes in certain routes which fall in the shortest path to the nodes also increases to next commercial diameter.

Table 4 provides the solutions obtained for selected weightage values namely $(0.8,0.2),(0.6,0.4),(0.4,0.6)$, and $(0.2,0.8)$. The first weightage belongs to cost and second to RM. When more weightage being allotted to normalized objective function of cost, the resulting solution has lesser cost and lesser RM values and vice versa. Table 4 shows that network configuration belongs to higher cost, and more RM value follows the same similar configuration and increase in the diameter takes place sequentially from the lower to higher cost solution. If more

Table 4 Optimal solutions for the network-based second objective function

\begin{tabular}{|c|c|c|c|c|c|c|c|c|}
\hline \multirow[t]{2}{*}{ Pipe ID } & \multicolumn{4}{|c|}{ Maximum diameter limited to $1016 \mathrm{~mm}$} & \multicolumn{4}{|c|}{ Maximum diameter limited to $1524 \mathrm{~mm}$} \\
\hline & $\begin{array}{l}W_{\mathrm{c}}=0.8 \\
W_{\mathrm{p}}=0.2 \\
\text { Solution 1 }\end{array}$ & $\begin{array}{l}W_{\mathrm{c}}=0.6 \\
W_{\mathrm{p}}=0.4 \\
\text { Solution } 2\end{array}$ & $\begin{array}{l}W_{\mathrm{c}}=0.4 \\
W_{\mathrm{p}}=0.6 \\
\text { Solution } 3\end{array}$ & $\begin{array}{l}W_{\mathrm{c}}=0.2 \\
W_{\mathrm{p}}=0.8 \\
\text { Solution } 4\end{array}$ & $\begin{array}{l}W_{\mathrm{c}}=0.8 \\
W_{\mathrm{p}}=0.2 \\
\text { Solution } 5\end{array}$ & $\begin{array}{l}W_{\mathrm{c}}=0.6 \\
W_{\mathrm{p}}=0.4 \\
\text { Solution } 6\end{array}$ & $\begin{array}{l}W_{\mathrm{c}}=0.4 \\
W_{\mathrm{p}}=0.6 \\
\text { Solution } 7\end{array}$ & $\begin{array}{l}W_{\mathrm{c}}=0.2 \\
W_{\mathrm{p}}=0.8 \\
\text { Solution } 8\end{array}$ \\
\hline 1 & 1016 & 1016 & 1016 & 1016 & 1524 & 1524 & 1524 & 1524 \\
\hline 2 & 1016 & 1016 & 1016 & 1016 & 1524 & 1524 & 1524 & 1524 \\
\hline 3 & 1016 & 1016 & 1016 & 1016 & 1270 & 1524 & 1524 & 1524 \\
\hline 4 & 1016 & 1016 & 1016 & 1016 & 1270 & 1524 & 1524 & 1524 \\
\hline 5 & 1016 & 1016 & 1016 & 1016 & 1016 & 1524 & 1524 & 1524 \\
\hline 6 & 1016 & 1016 & 1016 & 1016 & 1016 & 1270 & 1524 & 1524 \\
\hline 7 & 1016 & 1016 & 1016 & 1016 & 1016 & 1270 & 1270 & 1270 \\
\hline 8 & 1016 & 762 & 1016 & 1016 & 762 & 1270 & 1270 & 1270 \\
\hline 9 & 762 & 762 & 762 & 1016 & 762 & 1016 & 1270 & 1016 \\
\hline 10 & 762 & 762 & 762 & 1016 & 762 & 762 & 762 & 1016 \\
\hline 11 & 609.6 & 762 & 762 & 1016 & 609.6 & 609.6 & 762 & 762 \\
\hline 12 & 508 & 609.6 & 609.6 & 762 & 508 & 508 & 609.6 & 762 \\
\hline 13 & 304.8 & 508 & 609.6 & 609.6 & 304.8 & 609.6 & 762 & 304.8 \\
\hline 14 & 508 & 609.6 & 762 & 762 & 406.4 & 508 & 406.4 & 609.6 \\
\hline 15 & 609.6 & 762 & 762 & 1016 & 406.4 & 304.8 & 304.8 & 762 \\
\hline 16 & 1016 & 1016 & 1016 & 1016 & 762 & 304.8 & 304.8 & 1524 \\
\hline 17 & 1016 & 1016 & 1016 & 1016 & 762 & 508 & 609.6 & 1524 \\
\hline 18 & 1016 & 1016 & 1016 & 1016 & 1016 & 762 & 1016 & 1524 \\
\hline 19 & 1016 & 1016 & 1016 & 1016 & 1016 & 762 & 1016 & 1524 \\
\hline 20 & 1016 & 1016 & 1016 & 1016 & 1270 & 1524 & 1524 & 1524 \\
\hline 21 & 609.6 & 609.6 & 762 & 762 & 609.6 & 609.6 & 762 & 762 \\
\hline 22 & 406.4 & 406.4 & 508 & 508 & 304.8 & 406.4 & 406.4 & 508 \\
\hline 23 & 762 & 1016 & 1016 & 1016 & 1016 & 1270 & 1524 & 1016 \\
\hline 24 & 508 & 762 & 762 & 1016 & 762 & 1016 & 1016 & 609.6 \\
\hline 25 & 304.8 & 508 & 609.6 & 762 & 609.6 & 762 & 1016 & 304.8 \\
\hline 26 & 609.6 & 406.4 & 508 & 508 & 406.4 & 609.6 & 609.6 & 762 \\
\hline 27 & 762 & 609.6 & 762 & 762 & 406.4 & 304.8 & 406.4 & 1016 \\
\hline 28 & 762 & 762 & 762 & 1016 & 508 & 304.8 & 304.8 & 1270 \\
\hline 29 & 406.4 & 508 & 508 & 609.6 & 406.4 & 508 & 609.6 & 609.6 \\
\hline 30 & 304.8 & 406.4 & 406.4 & 304.8 & 304.8 & 406.4 & 609.6 & 406.4 \\
\hline 31 & 304.8 & 304.8 & 304.8 & 406.4 & 304.8 & 304.8 & 304.8 & 304.8 \\
\hline 32 & 406.4 & 304.8 & 406.4 & 406.4 & 508 & 304.8 & 304.8 & 406.4 \\
\hline 33 & 406.4 & 406.4 & 508 & 508 & 609.6 & 304.8 & 304.8 & 508 \\
\hline 34 & 609.6 & 609.6 & 762 & 762 & 609.6 & 609.6 & 609.6 & 762 \\
\hline RI & 0.2980 & 0.3281 & 0.3384 & 0.3487 & 0.7798 & 0.8460 & 0.8763 & 0.8969 \\
\hline Cost (\$) & $67,10,999$ & $74,17,236$ & $77,97,775$ & $86,81,431$ & $71,47,182$ & $80,22,887$ & $91,66,292$ & $106,60,762$ \\
\hline$d_{\mathrm{w}}$ & 712.04 & 762.55 & 792.09 & 848.79 & 746.30 & 804.90 & 886.86 & 997.00 \\
\hline
\end{tabular}


weightage is given to the minimization of cost rather than maximizing the performance, the network is not configured on the basis of shortest path to demand nodes. The solution obtained under weightage value of $(0.8,0.2)$ is observed distinct from remaining three solutions presented in Table 4. The similar observations have been made with other two RMs. When higher importance to the cost is given, the differential optimization algorithm searches a low-cost solution very close to minimum cost solution (optimal solution without RM) at the same time to have maximum performance within its boundary limits which ultimately configure the network without any shortest path logic. It is to be understood that the optimal solution without performance measure or performance constraints could result in distinct network pipe size configuration comparing any shortest path-based optimal approaches' solutions as it takes advantage of rounding to the commercial diameter which can provide some extra-annular to route the flow in multipath way.

The performance measure of the Hanoi network cannot be increased further as two of its pipes from the source have velocity greater than $6 \mathrm{~m} / \mathrm{s}$ which makes large pressure head loss in the network. The velocity of these pipes can be reduced only by increasing its size else parallel pipes need to be provided. Since optimization of the network needs to be done with the options tabulated in Table 1 according to Fujiwara and Khang (1990), all the researchers used same options and it is optimized. These two pipes are always assigned as $1016 \mathrm{~mm}$ diameter what so ever the cost of the network obtained through several approaches and algorithms. In this study, two more options have been added in the list. These two new pipes will have cost of 347.13 units and 458.2 units, respectively. The network is again optimized for revised set of pipe sizes for pressure head limit $30 \mathrm{~m}$. As two more options are incorporated in the optimization, the search space size has expanded and which warranted more number of generations. For revised options of diameter, population size of 40 and number of generation is fixed as 20,000 .

The minimum cost solution obtained for $30 \mathrm{~m}$ pressure head limit is $\$ 5,275,863$. It is to be noted that this cost is lesser than the minimum cost solution obtained while maximum diameter size is kept as $1016 \mathrm{~mm}$ for same pressure limit. RI, MRI, and PE values of minimum cost network are $0.364,0.849$, and 0.555 , respectively. It is very clear that though the cost of this network $(\$ 5,275,863)$ is lesser than optimal cost $(\$ 6,081,087)$ corresponding to maximum pipe diameter option $1016 \mathrm{~mm}$, respective performance measure values are higher. Hence, increasing pipe size options at higher side is justifiable. Table 4 shows the optimal solution obtained for four different combinations of weights adopted in the normalized objective function. It is to be noted that the network is configured along shortest path concept as similar to the previous case. In certain branches to the nodes, the telescopically decreasing pipes may not be the case due to selection of commercial diameters which will provide extra-annular area than actually required. This brings certain benefits to the network configuration in view of cost minimization. The water supposed to be delivered via shortest path to certain nodes will be directed along a path distinct from shortest path which may be longest, but that brings the cost economy in view of additional annular area available due to discrete selection of pipe sizes than actually required if continuous size is permitted. According to minimum spanning tree of Honai network, pipe identity numbers 14, 25 , and 31 are acting as the redundant members. The shortest path for node 13 falls in the route connecting pipe ID 1-12. Table 4 shows that all the presented solution here follows this path and pipe sizes are telescopically reducing along this path. For node ID 14, the shortest path from source is along pipe identity numbers 1, 2, 19, 18, 17, 16, 15, and 14. Except solution 7, remaining solutions fall in this path. In solution 7, pipes close to redundant pipe 14 is having higher diameter than its neighboring pipes, which indicate that the path selected for node 15 is $1-9,13$, and 14. But up to node 15, it follows along 1, 2, 19, 18, 17, 16, and 15. Similar observation can be made for node 26 , except solution 7 , the remaining solutions adhere to the same path in which size of the pipe is reducing telescopically. In case of node 31 , the shortest path appears along pipes ID numbers $1,2,20,23,24,25,34$, and 33. All the solution follows this path in conveying water to meet demand for node 31 . Hence, it is very clear from this analysis that water is transported along the shortest path when network is optimized for higher performance. Increase in the cost of network is attributed with an increase in weighted diameter. There is a drastic increase in the performance value when two higher diameter options are made in the search space. Selection of lower and upper bound in diameter options is another crucial factor in enhancing performance measures. It is to be noted that flows are usually concentrated on the shortest path to minimize the cost. However, this may not be true always (Maidamwar et al. 2000); for an example, in the case of layout and difference in elevations between the nodes along the path favors to take up circuitous route due to availability of gravity.

\section{Conclusion}

Getting an optimal design solution for water distribution network (WDN) is the most explored research area with a main objective of minimizing the cost of networks. The least cost design often fails under changes in nodal 
demands and pipe roughness. Reliability, robustness, or resilience measures are required to be considered simultaneously with economic cost in the optimal WDN design. Exact quantification of uncertainty in the demand and change in the roughness of the pipe due to age is normally addressed as a difficult task in the design. Hence, designing the network with some consideration to overcome these potential problems in terms of measures such as resilience or reliability is being worth adding design parameters. This paper attempts to minimize the cost of the network per resilience measure subject to pressure head constraint and limited to select within available commercial sizes of pipe. And this paper also presented the simple way to integrate the cost of the network and resilience measure as a single objective function through normalization approach and its capability is addressed using benchmark network. Honai network is considered to optimize the network with the proposed objective functions using differential evolution (DE) algorithm. In the present approach, the multi-objective nature of problem is converted into single objective (normalized objective function) and Pareto front is developed by changing the weights for both cost and resilience measure. Hence, it warranted several runs and trial runs in each selected weights. This also can be solved directly using non-dominated sorting approach which can produce Pareto front. It is evident from the study that whenever the performance measure is given prime importance than cost, then network's pipes are configured as telescopically decreasing size along shortest path. Although sizing the pipes for water distribution network based on shortest flow path results higher than the least cost solution (global least cost), analysis of results indicates that such a solution is found to be promising as far as resilience point of view. Handling two different units of objective functions as single objective formulation with one metric requires normalization. The proposed way of normalization and combining through weights provided an easy way for decision maker to select a best solution. Real water distribution network may contain a different set of objectives to address the different stakeholders concerns. Although considered objective functions cannot fully represent the complexity real system, proposed formulation provides an easy way of combining several objectives and generates the Pareto front with minimum number of solutions.

Open Access This article is distributed under the terms of the Creative Commons Attribution 4.0 International License (http:// creativecommons.org/licenses/by/4.0/), which permits unrestricted use, distribution, and reproduction in any medium, provided you give appropriate credit to the original author(s) and the source, provide a link to the Creative Commons license, and indicate if changes were made.

\section{References}

Bolognesi A, Bragalli C, Marchi A, Artina S (2014) Multiobjective design of water distribution networks through the generation of pseudofronts in the hydraulically feasible region. J Comput Civ Eng. doi:10.1061/(ASCE)CP.1943-5487.0000280

Bruneau M, Chang S, Eguchi R, Lee G, O'Rourke T, Reinhorn AM, Shinozuka M, Tierney K, Wallace W, Winterfelt DV (2003) A framework to quantitatively assess and enhance the seismic resilience of communities. Earthq Spectra 19(4):733-752

Cimellaro GP, Reinhorn AM, Bruneau M (2010) Framework for analytical quantification of disaster resilience. Eng Struct 32(11):3639-3649

Cimellaro GP, Tinebra A, Renschler C, Fragiadakis M (2015) New resilience index for urban water distribution networks. J Struct Eng ASCE. doi:10.1061/(ASCE)ST.1943-541X.0001433

Creaco E, Franchini M, Walski TM (2014) Accounting for phasing of construction within the design of water distribution networks. J Water Resour Plan Manag 140(5):598-606

Creaco E, Franchini M, Todini E (2016) The combined use of resilience and loop diameter uniformity as a good indirect measure of network reliability. Urban Water J. 13(2):167-181

Davis CA (2014) Water system service categories, post-earthquake interaction, and restoration strategies. Earthq Spectra 30(4):1487-1509

Dong X, Liu S, Tao T, Li S, Xin K (2012) A comparative study of differential evolution and genetic algorithms for optimizing the design of water distribution systems. J Zhejiang Univ Sci A (Applied Physics and Engineering) 13(a):674-686

Fu G, Kapelan Z, Reed P (2012) Reducing the complexity of multiobjective water distribution system optimization through global sensitivity analysis. J Water Resour Plan Manag 138(3): 196-207

Fujiwara O, Khang DB (1990) A two-phase decomposition method for optimal design of looped water distribution networks. Water Resour Res 26(4):539-549

Fujiwara O, Khang DB (1991) Correction to 'A two-phase decomposition method for optimal design of looped water distribution networks' by O. Fujiwara and D. B. Khang. Water Resour Res 27(5):985-986

Howard G, Bartram J (2010) The resilience of water supply and sanitation in the face of climate change, Vision 2030, Technical report, World Health Organization, Geneva, Switzerland

Jayaram N, Srinivasan K (2008) Performance-based optimal design and rehabilitation of water distribution networks using life cycle costing. Water Resour Res 44(W01417): 1417

Kadu MS, Gupta R, Bhave PR (2008) Optimal design of water networks using a modified genetic algorithm with reduction in search space. J Water Resour Plan Manag 134(2):147-160.4

Liu D, Chen X, Nakato T (2012) Resilience assessment of water resources system. Water Resour Manag. doi:10.1007/s11269012-0100-7

Loucks DP, Beek EV (2005) Water resources planning and management: model sensitivity and uncertainty analysis. UNESCO publishing, Paris, pp 255-290

Maidamwar P, Gupta R, Bhave PR (2000) Study of path concept for selecting tree in optimal design of looped water distribution systems. J Indian Water Works Assoc 32(1):29-34

Marchi A, Dandy G, Wilkins A, Rohrlach H (2014) Methodology for comparing evolutionary algorithms for optimization of water distribution systems. J Water Resour Plan Manag 140(1):22-31 
Ostfeld A, Oliker N, Salomons E (2014) Multiobjective optimization for least cost design and resiliency of water distribution systems. J Water Resourc Plan Manag 140(12):04014037-01-12

Pandit A, Crittenden JC (2012) Index of network resilience (INR) for urban water distribution systems. In: Proc. 2012 Critical Infrastructure Symposium, Arlington, VA

Piratla KR (2015) Investigation of sustainable and resilient design alternatives for water distribution networks. Urban Water. doi:10.1080/1573062X.2014.994001

Prasad TD, Park NS (2004) Multiobjective genetic algorithms for design of water distribution networks. J Water Resour Plan Manag 130(1):73-82

Rossman LA (2000) EPANET 2 users manual, National Risk Management Research Laboratory, Office of Research and Development, U.S. Environmental Protection Agency, Cincinnati, Ohio, USA

Saldarriaga JG, Ochoa S, Moreno ME, Romero N, Cortés OJ (2010) Prioritised rehabilitation of water distribution networks using dissipated power concept to reduce non-revenue water. Urban Water J 7(2):121-140

Savic DA, Walters GA (1997) Genetic algorithms for least cost design of water distribution networks. J Water Resour Plan Manag 123(2):67-77

Storn R, Price K (1995) Differential evolution-a simple and efficient adaptive scheme for global optimization over continuous spaces. Technical report, International Computer Science Institute, Berkeley, CA

Suribabu CR (2010) Differential evolution algorithm for optimal design of water distribution networks. J Hydroinf 12(1):66-82

Suribabu CR, Neelakantan TR (2012) Sizing of water distribution pipes based on performance measure and break-repair-replacement economics. ISH J Hydraul Eng 18(3):241-251

Suribabu CR, Prashanth K, Vignesh Kumar S, Sai Ganesh N (2016) Resilience enhancement methods for water distribution networks. Jordon J Civ Eng 10(2):216-231

Todini E (2000) Looped water distribution networks design using a resilience index based heuristic approach. Urban Water 2(2):115-122

Vasan A, Simonovic SP (2010) Optimization of water distribution network design using differential evolution. J Water Resour Plan Manag 136(2):279-287
Wang C, Blackmore J, Wang X, Yum KK, Zhou M, Diaper C, McGregor G, Anticev J (2009) Overview of resilience concepts, with application to water resource systems, eWater Cooperative Research Centre Technical report, eWater CRC, University of Canberra, Australia

Wang Q, Guidolin M, Savic D, Kapelan Z (2014) Two-objective design of benchmark problems of a water distribution system via MOEAs: towards the best known approximation of the true Pareto front. J Water Resour Plan Manag. doi:10.1061/ ASCE(WR).1943-5452.0000460

Wu W, Maier HR, Simpson AR (2011) Surplus power factor as s resilience measure for assessing hydraulic reliability in water transmission system optimization. J Water Resourc Plan Manag 137(6):542-546

Yazdani A, Jeffray P (2012) Applying network theory to quantify the redundancy and structural robustness of water distribution system. J Water Resour Plan Manag 138(2):153-161

Zheng F, Simpson AR, Zecchin AC (2011a) Performance study of differential evolution with various mutation strategies applied to water distribution system optimization World Environmental and Water Resources Congress 2011: Bearing Knowledge for Sustainability-Proceedings of the 2011 World Environmental and Water Resources Congress, pp 166-176

Zheng F, Simpson AR, Zecchin AC (2011b) A combined NLPdifferential evolution algorithm approach for the optimization of looped water distribution systems. Water Resour Res 47(8):W08531

Zheng F, Simpson AR, Zecchin AC (2012a) A performance comparison of differential evolution and genetic algorithm variants applied to water distribution system optimization, World Environmental and Water Resources Congress 2012: Crossing Boundaries, Proceedings of the 2012 Congress, pp 2954-2963

Zheng F, Zecchin AC, Simpson AR (2012b) Self-adaptive differential evolution algorithm applied to water distribution system optimization. J Comput Civ Eng 27(2):148-158 